\title{
Characteristics of fluid substitution in porous rocks
}

\author{
Li Shengjie* \\ School of Resource and Information Technology, China University of Petroleum, Beijing 102249, China \\ State Key Laboratory of Petroleum Resource and Prospecting, China University of Petroleum, Beijing 102249, China \\ Key Laboratory of CNPC Geophysical Exploration, China University of Petroleum, Beijing 102249, China
}

\begin{abstract}
Analysis of the effect of changes in fluid properties of rocks on the compressional-wave velocity $V_{\mathrm{P}}$ and shear-wave velocity $V_{\mathrm{S}}$ is very important for understanding the rock physical properties, especially in oilfield exploration and development. The fluid substitution process was analyzed by using ultrasonic measurement and theoretical calculations. The results showed that the effect of fluid substitution on the rock elastic modulus was mainly controlled by fluid properties, saturation, and confining pressure. For a rock with specific properties and porosity, the result of theoretical prediction for fluid substitution accorded with the experimental result under high confining pressure (higher than $60 \mathrm{MPa}$ for our experimental data), but failed to describe the trend of experimental result under low confining pressure and $V_{\mathrm{P}}$ predicted by Gassmann's equation was higher than that measured by experiment. A higher porosity resulted in stronger sensitivity of the bulk modulus of saturated rocks to the change of fluid properties.
\end{abstract}

Key words: Fluid substitution, velocity, bulk modulus, porosity, saturation

\section{Introduction}

The changes of pore fluid properties have a specific effect on the compressional-wave velocity $V_{\mathrm{P}}$ and shearwave velocity $V_{\mathrm{S}}$ of the fluid-saturated rocks. Quantitative study on the variation of $V_{\mathrm{P}}$ and $V_{\mathrm{S}}$ with fluid properties is very important for further investigation of rock properties, especially for hydrocarbon exploration and development (Wei et al, 2008; Di et al, 2006). It provides a basis for amplitudeversus-offset (AVO) analysis, 4-D seismic reservoir monitoring (Tsuneyama and Mavko, 2007) and seismic interpretation to identify and predict hydrocarbon.

The direct way to examine the effect of changes of pore fluid on the physical properties of rocks is laboratory measurement (Batzle et al, 2006; Best et al, 2007). Considerable research was done on the measurement of $V_{\mathrm{P}}$ and $V_{\mathrm{S}}$ of the saturated rocks, and the uncertainties of the experimental results were analyzed (Shi et al, 1998). However, there are relatively few reports of experimental results of fluid substitution in porous rocks. During fluid substitution, $V_{\mathrm{P}}$ and $V_{\mathrm{S}}$ of the saturated rocks are affected not only by the physical properties of pore fluid, but also by porosity (Dvorkin et al, 2007; Shapiro and Kaselow, 2005), pore shape, permeability, clay content of rocks (Berryman, 2005) and the physical environment (such as in-situ pressure and temperature) (Gurevich, 2004; Kozlov, 2004; Wang, 2001). Generally, the physical properties of porous rocks are related to the characteristics of pore fluid through the BiotGassmann model. Under a specific condition, the validity of this model has been proved by many authors through

*Corresponding author. email: richard@cup.edu.cn

Received October 28, 2008 laboratory measurements (Wang, 2001). Wang (2001) discussed the basic assumption and application range of this model in detail.

Based on the Biot-Gassmann fluid substitution model, we presented an explicit equation that can describe the relationship between the change rate of bulk modulus of saturated rocks and that of pore fluid. Laboratory measurements of oil/water substitution were carried out and the experimental results were analyzed according to the relationship. Finally, the factors which affected the fluid substitution were discussed in depth.

\section{Poroelastic theory}

Reservoir rocks are porous aggregates consisting of various mineral grains, and the pore space may be filled with one or more fluids. When a seismic wave propagates to the reservoir, it will compress the porous rocks, so the mineral framework of rocks is deformed. The pore fluid is compressed, and an increment of pore pressure, which resists the compression and thus stiffens the rock, is induced. The Biot-Gassmann theory provides the general relationship between velocity and elastic modulus (Han and Batzle, 2004), and the velocity of porous medium can be expressed as follows:

$$
\begin{aligned}
& \rho V_{\mathrm{p}}^{2}=K_{\mathrm{b}}+K_{\mathrm{p}}+\frac{4}{3} \mu \\
& \rho V_{\mathrm{s}}^{2}=\mu
\end{aligned}
$$

where $V_{\mathrm{P}}$ and $V_{\mathrm{S}}$ are, respectively, the velocities of compressional wave and shear wave; $K_{\mathrm{b}}$ and $\mu$ are, respectively, the bulk modulus and shear modulus of the grain framework. Assuming the surface of the grains is sufficiently 
wetted by the fluid, the modulus of framework is independent of fluid saturation. For a specific kind of mineral grains, $K_{\mathrm{b}}$ depends on the properties of mineral, and varies greatly with porosity. $\rho$ is bulk density of porous medium. $K_{\mathrm{p}}$ is the pore bulk modulus (Murphy et al, 1993), which is defined as:

$$
K_{\mathrm{p}}=K_{\mathrm{s}} \frac{\left(1-K_{\mathrm{b}} / K_{\mathrm{s}}\right)^{2}}{1-K_{\mathrm{b}} / K_{\mathrm{s}}+\phi\left(K_{\mathrm{s}} / K_{\mathrm{f}}-1\right)}
$$

where $K_{\mathrm{s}}$ and $K_{\mathrm{f}}$ are, respectively, the bulk moduli of the mineral and the pore fluid. $\phi$ is the porosity of porous rocks.

The mineral bulk modulus $K_{\mathrm{s}}$ is calculated by VoigtReuss-Hill averaging of the bulk moduli of all kinds of minerals that compose the rock, which is expressed as:

$$
K_{\mathrm{s}}=\frac{1}{2}\left(\sum_{i=1}^{n} c_{i} K_{i}+\sum_{i=1}^{n} c_{i} / K_{i}\right)
$$

where, $c_{i}$ and $K_{i}$ are the bulk coefficient and modulus of the $i$ th mineral component, respectively.

The bulk modulus of framework $K_{\mathrm{b}}$ can be obtained from the laboratory measured velocity and density. Mavko (Mavko et al, 1995) gave an improved method to determine $K_{\mathrm{b}}$.

For a dry rock, the relationship between bulk modulus of framework and mineral is given as follows:

$$
K_{\mathrm{b}}=K_{\mathrm{s}}(1-\beta)
$$

where $\beta$ is sometimes called Biot coefficient (Olsen et al, 2008 ), which is defined as the ratio of the change of pore volume $\Delta v_{\mathrm{p}}$ to the change of total volume $\Delta v$ at the same pore pressure.

$$
\beta=\frac{\Delta v_{\mathrm{p}}}{\Delta v}=1-\frac{K_{\mathrm{b}}}{K_{\mathrm{s}}}
$$

For single phase fluid, Eq. (2) provides a complete solution for the bulk modulus of the saturated rock. $K_{\mathrm{f}}$ and $\rho_{\mathrm{f}}$ can be calculated by using Wood's equation (Murphy et al, 1993):

$$
\begin{aligned}
& \frac{1}{K_{\mathrm{f}}}=\sum \frac{S_{i}}{K_{i}} \\
& \rho_{\mathrm{f}}=\sum S_{i} \rho_{i}
\end{aligned}
$$

where $K_{\mathrm{f}}$ is the bulk modulus of the mixed fluid, $\rho_{\mathrm{f}}$ is the density of the mixed fluid, $K_{i}$ and $\rho_{i}$ are the bulk modulus and the density of the $i$ th phase fluid. $S_{i}$ is the saturation expressed by volume fraction of the pore space.

According to Eq. (2), the effect of pore fluid on the physical properties of saturated rocks is shown by the change of pore bulk modulus. For saturated rocks, a change in pore fluid properties will result in the change of bulk modulus of the saturated rock. The rate of change of the bulk modulus can be given by (Li, 2005):

$$
\frac{\partial K}{K}=\left(\frac{\phi}{\left(1-K_{\mathrm{b}} / K_{\mathrm{s}}\right)^{2}} \cdot \frac{K_{\mathrm{p}}}{K} \cdot \frac{K_{\mathrm{p} 2}}{K_{\mathrm{f} 2}}\right) \cdot \frac{\partial K_{\mathrm{f}}}{K_{\mathrm{f}}}
$$

Eq. (6) can be rewritten in the following form:

$$
\frac{\partial K}{K}=c \cdot \frac{\partial K_{\mathrm{f}}}{K_{\mathrm{f}}}
$$

where

$$
\begin{aligned}
& c=\left(\frac{\phi}{\left(1-K_{\mathrm{b}} / K_{\mathrm{s}}\right)^{2}} \cdot a \cdot b\right) \\
& a=\frac{K_{\mathrm{p}}}{K} \\
& b=\frac{K_{\mathrm{p}}}{K_{\mathrm{f}}}
\end{aligned}
$$

Here, $a, b$ and $c$ are coefficients that describe the change rate of bulk modulus of the saturated rocks varying with the change rate of bulk modulus of different pore fluids. Eq. (7) quantitatively shows the effect of the change of pore fluid bulk modulus on the saturated rock bulk modulus. The sensitivity of the bulk modulus of different porous media during fluid substitution for different rocks can be obtained using Eq. (7).

\section{Experimental study}

Laboratory measurements of core samples provide important reservoir rock data. Systematic laboratory studies on fluid substitution in relation to rock properties are the basis for understanding the relationship between seismic velocity and rock properties such as fluid properties, saturation, and porosity.

In order to analyze the validity of Eq. (7), measurements of $V_{\mathrm{P}}$ and $V_{\mathrm{S}}$ were made on a total of 32 samples at room temperature with the confining pressure of 10, 20, 40, 60, 80, 100 and $120 \mathrm{MPa}$ respectively. These samples measured in this experiment were sandstones coming from the Junggar Basin, Xinjiang, west China. Among these samples, 10 samples were dry and fully oil-saturated, and other 22 samples had different partial oil saturations. Standard ultrasonic technique was used to measure $V_{\mathrm{P}}$ and $V_{\mathrm{S}}$ of these samples. 


\subsection{Experimental procedure}

The samples were prepared as cylinders so that the wave path could be measured accurately. The two end-surfaces of the cylindrical samples had to be ground to flatness and parallel so that the dimensions and volume could be measured accurately.

The velocities of samples were measured by using the pulse transmission method. In this method, two piezoelectric transducers operating at a central frequency of $0.8 \mathrm{MHz}$ were positioned on the two parallel sides of the sample. Ultrasonic pulses generated by the pulse generator were converted to mechanical vibration by the transmitting piezoelectric transducer. After traveling through the rock sample, the mechanical (elastic) waves were converted back to an electrical signal by the receiving transducer. The travel time of the acoustic wave was measured by a digital oscilloscope and then the velocity was calculated by:

$$
V=\frac{L}{\Delta t}
$$

where, $V$ was the velocity of either the compressional wave or shear wave, $L$ was the sample length, and $\Delta t$ was the travel time in the sample.

Data acquisition was controlled by computer. Before testing, the operation of the test apparatus was verified by using an aluminum sample with known elastic properties. In the laboratory measurements, the sample was placed between two end caps. Isolation of the test samples from the confining fluid was achieved by jacketing. The jacketing was then secured to the end caps with a wire tie. Pore fluid outlets were left open, allowing a drained test. The sample was then sealed within the pressure vessel. Hydrostatic pressure was applied at the rate of $3 \mathrm{MPa} / \mathrm{min}$. After achieving hydrostatic conditions, data were recorded during the hydrostatic loading cycles. All tests were conducted at room temperature.

Before the ultrasonic measurements, the samples were cleaned using methanol to extract the oil and salt for preventing thermo-fracturing of the samples.

Samples were dried for 24 hours in an oven kept at $100{ }^{\circ} \mathrm{C}$, then transferred to a dry container, and finally taken as the dry samples. The fully oil-saturated samples were prepared by immersion in oil and vacuum pumping until no air bubble escaped.

Ultrasonic velocity measurements were made firstly on the dry sample after the weight of the dry samples was recorded. Using the method above mentioned, the dry sample was fully saturated, and at the same time, its weight was recorded. Therefore, the weight of oil can be obtained from the difference between the weight of the dry sample and that of the fully oil-saturated sample. Furthermore, the volume of oil can be calculated from oil density. Finally, the fully oilsaturated sample was placed in the pressure vessel.

To simulate fluid substitution, brine was injected into the oil-saturated sample on one side to displace the oil, and at the same time the oil discharged from the other side was collected. The volume of brine injected into the sample was approximately equal to the volume of oil discharged from the sample. Therefore, oil saturation $S_{\mathrm{o}}$ can be obtained from the volume of total oil and the volume of oil discharged from the sample. Ultrasonic velocity measurements were made on samples with different $S_{\mathrm{o}}$ and the samples were also weighed before and after the velocity measurements.

\subsection{Experimental results}

The results of the measured $V_{\mathrm{P}}$ and $V_{\mathrm{S}}$ of the dry samples are plotted in Fig. 1. Thin-section analysis of the samples used in this experiment showed that the sandstone samples were composed mainly of quartz, feldspar and clastic tuff fragments. Fig. 1 shows the variation of $V_{\mathrm{P}}$ and $V_{\mathrm{S}}$ of the dry samples under different confining pressures. $V_{\mathrm{P}}$ and $V_{\mathrm{S}}$ exhibit a strong correlation, that $V_{\mathrm{P}}$ and $V_{\mathrm{S}}$ increase as the confining pressure increases. The variation range of velocity gradually decreases with increasing confining pressure. In a specific range of confining pressure, continuing to increase the confining pressure has little influence on the velocity variation.

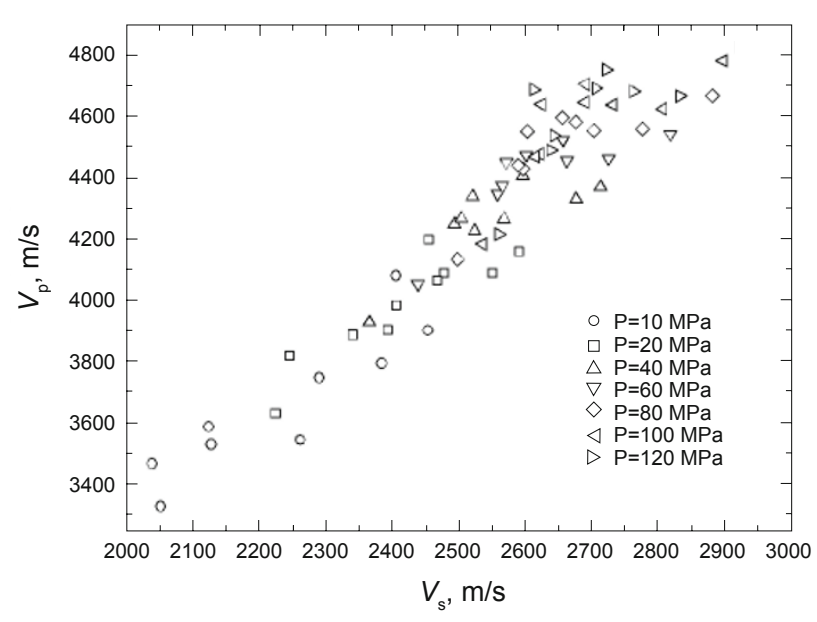

Fig. 1 Relation between $V_{\mathrm{P}}$ and $V_{\mathrm{S}}$ under different confining pressures for dry samples

In general, with increasing confining pressure, the applied stress leads to closed microcracks in the sample, lower porosity and larger contact area of rock grains. As a result, the measured ultrasonic velocity will increase. The average ratio of $V_{\mathrm{P}}$ to $V_{\mathrm{S}}$ is approximately equal to 1.65 .

When the dry sample was saturated with oil, $V_{\mathrm{P}}$ increased noticeably, but $V_{\mathrm{S}}$ increased only slightly, because of decreasing compressibility of the oil-saturated sample. The results of ultrasonic velocity measurements of the oil-saturated samples are shown in Fig. 2. The samples corresponding to the observation points in Fig. 2 are the same as the samples corresponding to the observation points in Fig. 1. The relationship of measured $V_{\mathrm{P}}$ and $V_{\mathrm{S}}$ shows a specific linear relation, with the correlation coefficient $r^{2}$ $=0.79$. However, the points in Fig. 2 are somewhat dispersed. The effect of the confining pressure on the oil-saturated samples is the same as that on the dry samples, but the range of the velocity variation with increasing confining pressure, compared with the dry samples, is reduced as a result of decreasing compressibility of the oil-saturated samples. For this reason, the relationship between $V_{\mathrm{P}}$ and $V_{\mathrm{S}}$ of the oilsaturated samples shows a strong linear relation in Fig. 2. 


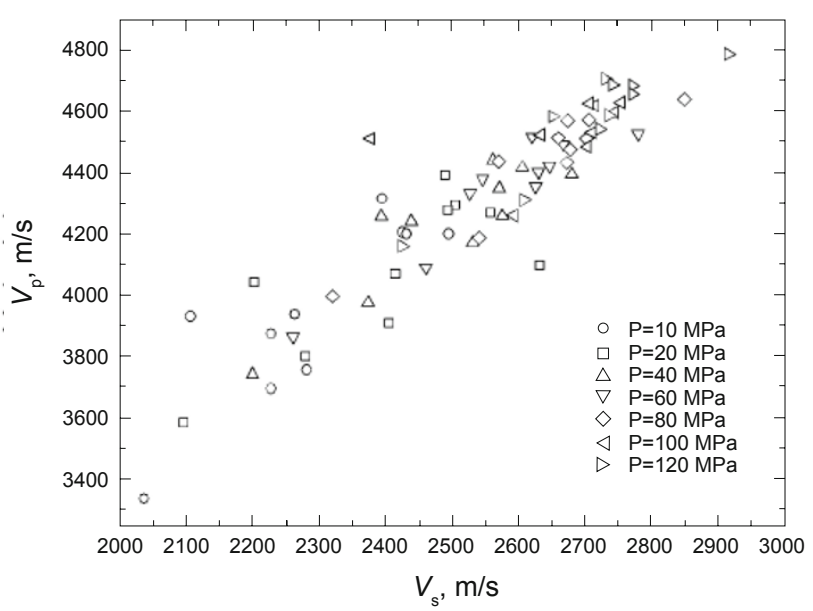

Fig. 2 Relation between $V_{\mathrm{P}}$ and $V_{\mathrm{S}}$ under different confining pressures for fully oil-saturated samples

The experiments of fluid substitution were performed on 22 samples at room temperature and different confining pressures. The results of measured velocity for the partially oil-saturated samples are plotted in Fig. 3, together with the corresponding fitting lines at each confining pressure with the average correlation coefficient $r^{2}=0.65$. The fitting lines were obtained by using the least-square polynomial fitting method.

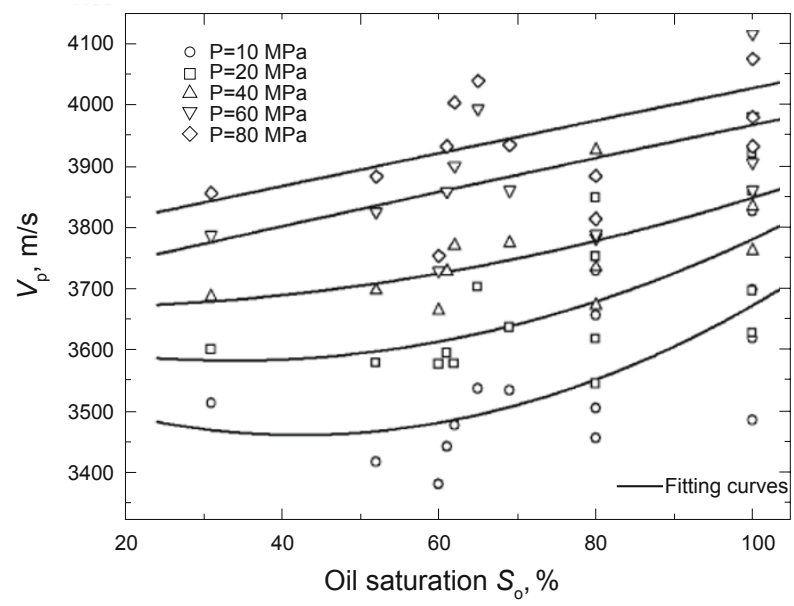

Fig. 3 Relation between $V_{\mathrm{P}}$ and oil saturation at different confining pressures

Although the correlations of $V_{\mathrm{P}}$ and $V_{\mathrm{S}}$ are poor, the trend of fitting lines roughly expresses the influence of the change in fluid properties on the velocities of oil-saturated samples during the fluid substitution. The change in fluid properties has only a little influence on $V_{\mathrm{S}}$, so the measured $V_{\mathrm{P}}$ is mainly discussed in the following section.

Fig. 3 shows that $V_{\mathrm{P}}$ of the oil-saturated samples decreases as $S_{\mathrm{o}}$ decreases and $V_{\mathrm{P}}$ increases as the confining pressure increases after oil is substituted by water. The effect of the confining pressure on the velocity of oil-saturated samples decreases with increasing pressures, as shown in Fig. 3. During the fluid substitution, the effect of $S_{\mathrm{o}}$ on $V_{\mathrm{P}}$ is affected by the confining pressure. The sensitivity of the velocity of saturated samples to $S_{\mathrm{o}}$ at a high confining pressure is larger than that at a low confining pressure.

\section{Interpretation}

The change of $V_{\mathrm{P}}$ with the variation of oil saturation can be quantified by the variation of fluid modulus. Given the frame bulk modulus, mineral bulk modulus, original pore fluid modulus, new pore fluid modulus, porosity and saturation, the effect of the fluid substitution on velocity can be simulated numerically according to Eq. (1) and (7).

\subsection{Effect of fluid modulus}

Based on the thin-section analysis of the samples and the measured velocity and density, the experimental results were analyzed theoretically in terms of Eq. (7). $K_{\mathrm{s}}$ is calculated from the sandstone's components using Eq. (3), with $K_{\mathrm{s}}=40.58 \mathrm{GPa} . K_{\mathrm{b}}$ and $\mu$ are obtained from the measured velocities of the dry samples under different confining pressures using Eq. (1). The bulk modulus of oil is determined by rearranging Eq. (2):

$$
K_{\mathrm{f}}=\frac{K_{\mathrm{s}}\left(K-K_{\mathrm{b}}\right) \phi}{K_{\mathrm{s}}\left(1-K_{\mathrm{b}} / K_{\mathrm{s}}\right)^{2}-\left(K-K_{\mathrm{b}}\right)\left(1-\phi-K_{\mathrm{b}} / K_{\mathrm{s}}\right)}
$$

The average porosity of samples is 0.26 . The bulk modulus of the substitution fluid (water) is $2.2 \mathrm{GPa}$, and the mixed fluid modulus is calculated by using Eq. (5).

The effect of fluid substitution on $V_{\mathrm{P}}$ can be analyzed by investigating the relationship between fluid modulus and bulk modulus of saturated rocks. It is clear, based on Eq. (1), that the bigger the change rate of the bulk modulus of saturated rocks, the more remarkable the variation of velocity caused by the change of fluid modulus. To analyze the effect of fluid substitution on saturated rock velocity, various parameters in fluid substitution are obtained by using Eqs. (1), (2) and (7), as shown in Fig. 4.

Fig. 4 shows the relationship among $\partial K / K, a, b, c$, and $\partial K_{\mathrm{f}} / K_{\mathrm{r}}$, with $K=20.72 \mathrm{GPa}, K_{\mathrm{f}}=3.54 \mathrm{GPa}$ and $K_{\mathrm{p}}=5.92 \mathrm{GPa}$.

For our experimental results, $\partial K / K, \partial K_{\mathrm{f}} / K_{\mathrm{f}}$, and $\partial K_{\mathrm{p}} / K_{\mathrm{p}}$ (the change rate of the bulk modulus) increase as fluid modulus $K_{\mathrm{f}}$ increases. The increment of $\partial K_{\mathrm{f}} / K_{\mathrm{f}}$ is the largest and that of $\partial K / K$ is the smallest. The relationship among $\partial K / K, \partial K_{\mathrm{p}} / K_{\mathrm{p}}$, and ${ }^{\partial K_{\mathrm{f}}} / K_{\mathrm{f}}$ is dominated by the coefficient curves $b$ and $c$ in Fig. 4(a). The curve $a$ exhibits the relationship between pore fluid and rock properties. It is the most important factor that affects the process of fluid substitution. Actually, for our samples, the coefficients $a, b$ and $c$ are roughly independent of the fluid modulus.

The change in fluid modulus depends on the change in $S_{\mathrm{o}}$. Therefore, the bulk modulus of oil saturated samples changes with $S_{\mathrm{o}}$ during the fluid substitution. Fig. 4(b) shows the relationship among $K_{\mathrm{f}}, \partial K / K, \partial K_{\mathrm{f}} / K_{\mathrm{f}}$, and $S_{0}$, reflects a quantitative relationship between $K_{\mathrm{f}}$ and $S_{\mathrm{o}}$, and directly quantifies the change of the bulk modulus of saturated 


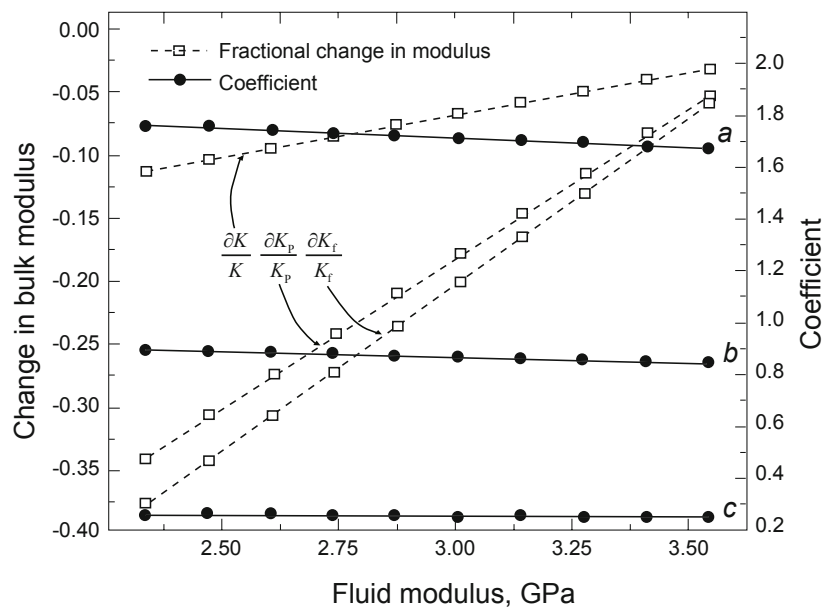

(a)

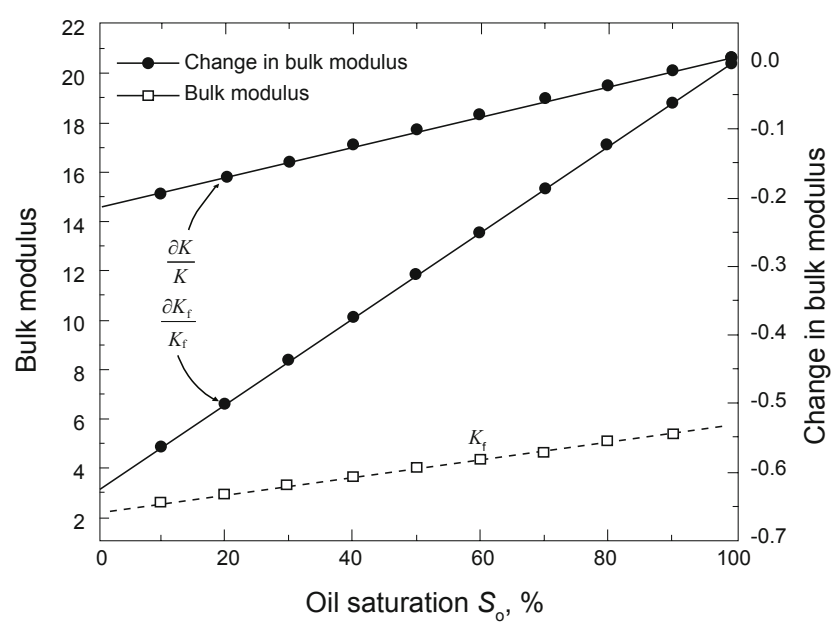

(b)

Fig. 4 Variation of bulk modulus of saturated rocks with different fluid properties (a) effect of change in fluid modulus, (b) effect of oil saturation

samples due to the change of $S_{0}$. It provides a convenient tool for estimating the seismic responses (such as velocity) resulting from the change of pore fluid properties of rocks.

\subsection{Theoretical prediction}

The results of the bulk modulus and velocity predicted by Eqs. (1) and (7) by using the parameters above mentioned are plotted in Fig. 5, where $K$ and $V_{\mathrm{P}}$ change with $S_{0}$, and the solid lines denote the bulk modulus or velocity.

Fig. 5(a) shows the comparison of the predicted velocity (solid line) and the measured velocity (scattered points), where the trend of predicted $V_{\mathrm{P}}$ changing with $S_{\mathrm{o}}$ is the same as that of measured $V_{\mathrm{P}}$. The predicted $V_{\mathrm{P}}$ agrees well with the measured $V_{\mathrm{P}}$ under the confining pressure of 60 $\mathrm{MPa}$. However, the predicted $V_{\mathrm{P}}$ is obviously greater than the measured $V_{\mathrm{P}}$ under the confining pressure of $10 \mathrm{MPa}$. It can be concluded that the effect of fluid substitution on $V_{\mathrm{P}}$ relates not only to $S_{\mathrm{o}}$ but also to the confining pressure. The difference between predicted and measured velocity is reduced as the confining pressure increases.
In Fig. 5(b), the predicted oil-saturated bulk modulus (solid line) according to Eq. (7) agrees well with the measured bulk modulus calculated from the measured velocity and density. The theoretical calculation shows that the predicted bulk modulus increases as $S_{\mathrm{o}}$ increases. The results predicted by theoretical calculation agree well with the corresponding results measured in laboratory, as shown in Fig. 5(a). It is concluded that the prediction according to Eq. (7) works very well in our experiment.

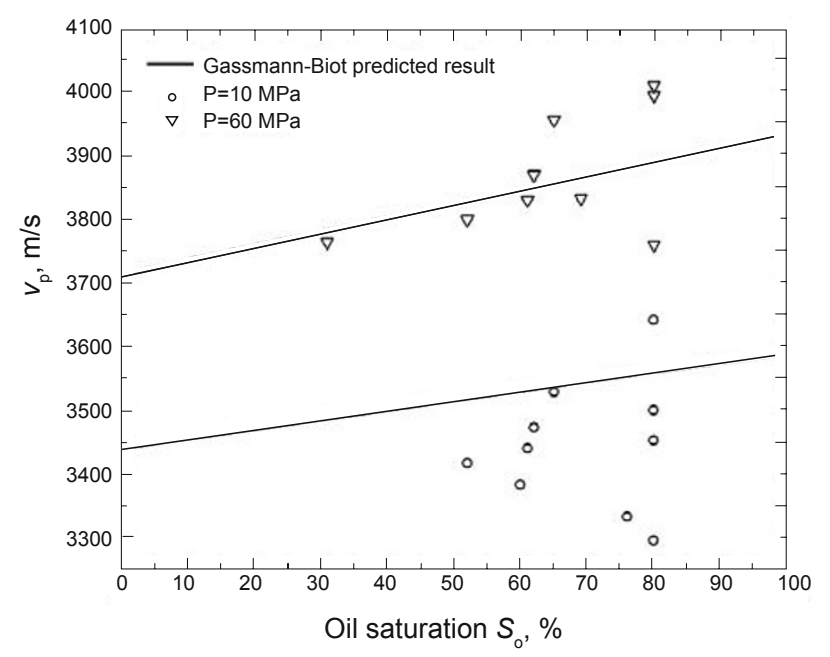

(a)

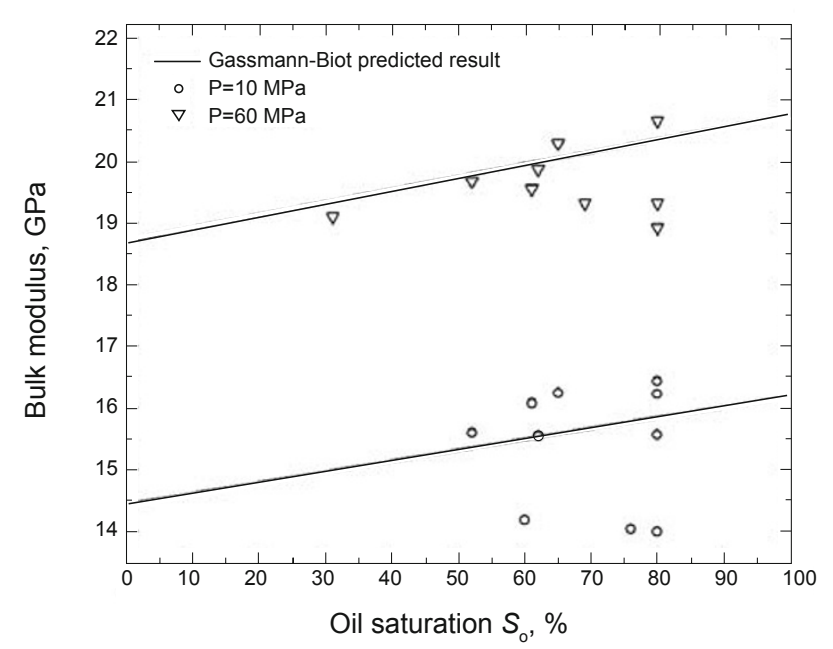

(b)

Fig. 5 Comparison of laboratory measurements with calculated results for different oil-saturated sandstones. (a) $V_{\mathrm{p}}$ versus $S_{\mathrm{o}}$, (b) bulk modulus versus $S_{\mathrm{o}}$

\section{Discussion}

The experimental and theoretical results showed that a change of bulk modulus of oil-saturated rocks depended not only on a change of fluid modulus but also on the confining pressure. Increasing confining pressure led to a change of grain contact, decrease of microcracks, and low 
compressibility of rocks. Therefore, it was important to investigate the effects of porosity on the process of fluid substitution according to Eq. (7).

The coefficient between $\partial K / K, \partial K_{\mathrm{f}} / K_{\mathrm{f}}$ in Eq. (7) is composed of the changes of fluid properties, pore modulus, and porosity. The effects of the coefficients $a$ and $b$ on the fluid substitution were dominated by pore bulk modulus and fluid modulus. The coefficient $c$ acted as a weight factor during the fluid substitution. Besides the effects of coefficient $a$ and $b, c$ was dominated by porosity $\phi, K_{\mathrm{s}}$, and $K_{\mathrm{b}}$. It could be further separated into two terms, that is, the term $\phi$ and the term $\beta$. Theoretically, both $\phi$ and $\beta$ could be set to be from 0 to 1 . In practice, the porosity is often set to be $0 \leq \phi \leq \phi_{c}$, where $\phi_{\mathrm{c}}$ is the critical porosity.

Fig. 6 shows a plot of the effect of the porosity on the process of fluid substitution. It is clear that during the fluid substitution, the increment of $\partial K / K$ decreases as the porosity increases or the bulk modulus of rocks decreases with increasing porosity. This relation indicates that the sensitivity of the bulk modulus of saturated samples to the fluid modulus decreases with increasing porosity when the fluid modulus is relatively low ( $<4.75 \mathrm{GPa}$, for this case), and is almost not affected by porosity when the fluid modulus is very large. In other words, $V_{\mathrm{P}}$ decreases with increasing porosity, and the sensitivity of $V_{\mathrm{P}}$ to the change of fluid properties is high when the fluid modulus is small at high porosity.

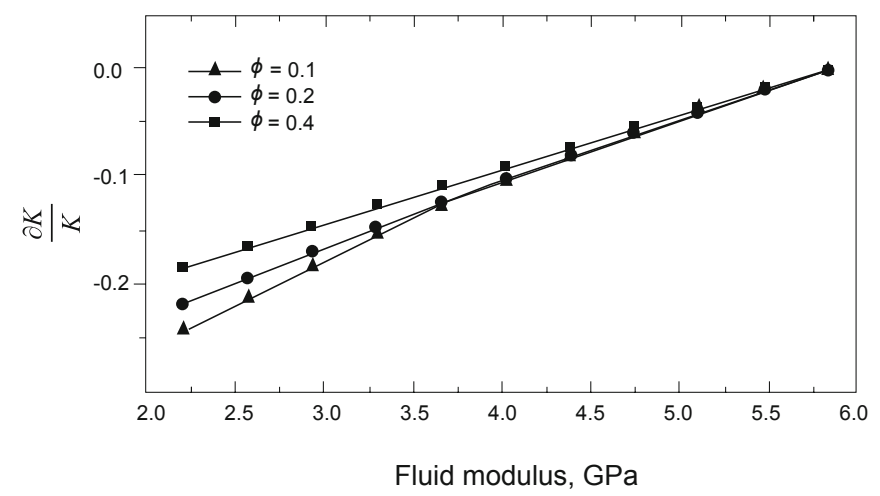

Fig. 6 Effect of porosity on the fluid substitution

According to Eq. (8), the coefficient $c$ is larger as the frame modulus of rocks is close to the mineral bulk modulus, that is, $K_{\mathrm{b}} \rightarrow K_{\mathrm{s}}$, or $\beta \rightarrow 0$. It is clear that $\partial K / K$ increases for rock with frame bulk modulus approaching zero, that is, $K_{\mathrm{b}} \rightarrow 0$, which is corresponding to 'soft' rocks. Therefore, the effect of the change of fluid properties for 'soft' rocks on elastic modulus is stronger than that for 'hard' rocks.

Eq. (7) presented here is important for fluid substitution in both theoretical analysis and laboratory simulation. This equation not only helps to analyze the experimental results of the fluid substitution, but also can be applied to 4-D seismic reservoir monitoring for quantitatively predicting reservoir fluids. A relationship between the bulk modulus of saturated rocks and fluid modulus can be quantified by Eq. (7) for a specific kind of rock and pore fluid, as shown in Fig. 4. It provides a convenient tool for analyzing and predicting the change of bulk modulus resulting from the change of pore fluid properties.

\section{Conclusions}

The experiment of fluid substitution was carried out by using the ultrasonic measurement under different confining pressures. The velocities of the partially saturated samples were measured during the fluid substitution. The experimental results showed that the effect of fluid substitution on rock elastic modulus was mainly controlled by fluid properties, saturation, and confining pressure. To quantitatively describe the process of fluid substitution, an expression was derived based on Gassmann's equation. The coefficient $c$ in Eq. (7) was the direct link between fluid modulus and bulk modulus of saturated rocks and was therefore the decisive factor of fluid substitution. Experimental results were theoretically analyzed by using Eq. (7). It can be concluded that, for a rock with fixed frame modulus and porosity, the result of theoretical prediction for fluid substitution accorded with the experimental result under high confining pressure (for our experimental data, the pressure is higher than $60 \mathrm{MPa}$ ). A higher porosity resulted in stronger sensitivity of the bulk modulus of saturated rocks to the change of fluid properties. Eq. (7) presented here is important for fluid substitution in both theoretical calculation and laboratory analysis.

\section{Acknowledgements}

This work was supported by the National Basic Research Program of China (Grant No.2007CB209601).

\section{References}

Batzle M L, Han D H and Hofmann R. Fluid mobility and frequencydependent seismic velocity — Direct measurements. Geophysics. 2006. 71(1): N1-N9

Berryman J G. Fluid effects on shear waves in finely layered porous media. Geophysics. 2005. 70(2): N1-N15

Best A I, Sothcott J and McCann C. A laboratory study of seismic velocity and attenuation anisotropy in near-surface sedimentary rocks. Geophysical Prospecting. 2007. 55(5): 609-625

Di B R, Wei J X and Mou Y G. Seismic physical modeling technology and its applications. Petroleum Science. 2006. 3(2): 39-46

Dvorkin J, Mavko G and Gurevich B. Fluid substitution in shaley sediment using effective porosity. Geophysics. 2007. 72(3): O1-O8

Gurevich B. A simple derivation of the effective stress coefficient for seismic velocities in porous rocks. Geophysics. 2004. 69(2): 393-397

Han D H and Batzle M L. Gassmann's equation and fluid-saturation effects on seismic velocities. Geophysics. 2004. 60(2): 398-405

Kozlov E A. Pressure-dependent seismic response of fractured rock. Geophysics. 2004. 69(4): 885-897

Li S J. Influence of lithology, porosity and variation of pore fluid saturation on rock elastic property. Oil and Gas Geology. 2005. 26(6): 760-764 (in Chinese)

Mavko G, Chan C and Mukerji T. Fluid substitutions: Estimating changes in $V_{\mathrm{P}}$ without knowing $V_{\mathrm{S}}$. Geophysics. 1995. 60(6): 17501755

Murphy W, Reische A and Hsu K. Modulus decomposition of 
compressional and shear velocity in sand bodies. Geophysics. 1993. 58(2): 227-239

Olsen C, Hedegaard K, Fabricius I L, et al. Prediction of Biot's coefficient from rock-physical modeling of North Sea chalk. Geophysics. 2008. 73(2): E89-E96

Shapiro S A and Kaselow A. Porosity and elastic anisotropy of rocks under tectonic stress and pore-pressure changes. Geophysics. 2005. 70(5): N27-N38

Shi X J, Xia C J and Wu Y G. The laboratory study on wave velocity under reservoir condition and its affection factors. Chinese Journal of
Geophysics. 1998. 41(1): 99-108 (in Chinese)

Tsuneyama F and Mavko G. Quantitative detection of fluid distribution using time-lapse seismic. Geophysical Prospecting. 2007. 55(2): 169184

Wang Z Z. Fundamentals of seismic rock physics. Geophysics. 2001. 66(2): 398-412

Wei J X, Di B R and Wang Q. Experimental study on the effect of fracture scale on seismic wave characteristics. Petroleum Science. 2008. 5(2): 119-125

(Edited by Hao Jie) 